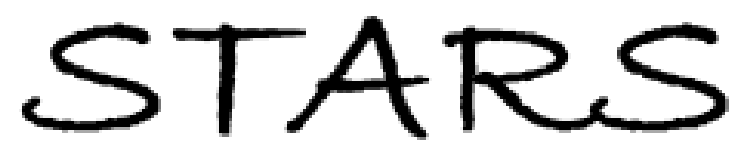

University of Central Florida

STARS

$1-1-2004$

\title{
Photonic switching based on the photoinduced birefringence in bacteriorhodopsin films
}

\author{
Yuhua Huang \\ University of Central Florida \\ Shin-Tson Wu \\ University of Central Florida \\ Youyuan Zhao
}

Find similar works at: https://stars.library.ucf.edu/facultybib2000 University of Central Florida Libraries http://library.ucf.edu

This Article is brought to you for free and open access by the Faculty Bibliography at STARS. It has been accepted for inclusion in Faculty Bibliography 2000 s by an authorized administrator of STARS. For more information, please contactSTARS@ucf.edu.

\section{Recommended Citation}

Huang, Yuhua; Wu, Shin-Tson; and Zhao, Youyuan, "Photonic switching based on the photoinduced birefringence in bacteriorhodopsin films" (2004). Faculty Bibliography 2000s. 4443.

https://stars.library.ucf.edu/facultybib2000/4443

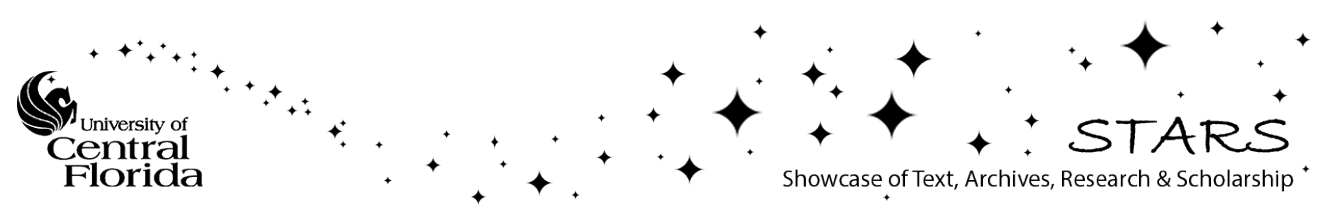




\section{Photonic switching based on the photoinduced birefringence in bacteriorhodopsin films}

Cite as: Appl. Phys. Lett. 84, 2028 (2004); https://doi.org/10.1063/1.1688456

Submitted: 08 September 2003 . Accepted: 26 January 2004 . Published Online: 16 March 2004

Yuhua Huang, Shin-Tson Wu, and Youyuan Zhao

ARTICLES YOU MAY BE INTERESTED IN

Enhancement of photoinduced anisotropy and all-optical switching in Bacteriorhodopsin films Applied Physics Letters 81, 3888 (2002); https://doi.org/10.1063/1.1521581

Applied Physics Letters

Mid-IR and THz frequency combs special collection

Read Now! 


\title{
Photonic switching based on the photoinduced birefringence in bacteriorhodopsin films
}

\author{
Yuhua Huang and Shin-Tson $\mathrm{Wu}^{\mathrm{a})}$ \\ School of Optics, University of Central Florida, Orlando, Florida 32816 \\ Youyuan Zhao \\ Department of Optics and Engineering, Fudan University, Shanghai, 200433, People's Republic of China
}

(Received 8 September 2003; accepted 26 January 2004)

\begin{abstract}
Photoinduced birefringence in bacteriorhodopsin films was investigated using pump-probe method and its application for photonic switching explored. A diode-pumped second-harmonic YAG laser was used as a pumping beam and a diode laser at $\lambda=660 \mathrm{~nm}$ was used as a probing beam. The pump and probe beams overlap at the sample. Without the pumping beam, the probing light cannot transmit the analyzer to the detector. However, due to the photoinduced anisotropy, a portion of the probing light is detected when the pumping beam is present. Since $\lambda=660 \mathrm{~nm}$ is far from the absorption peak $(\sim 570 \mathrm{~nm})$ of the ground state, the photoinduced birefringence predominates. Using the intensity-dependent photoinduced birefringence in a bacteriorhodopsin film, we have demonstrated a photonic switch with $\sim 1000: 1$ contrast ratio, $\sim 0.6 \mathrm{~s}$ rise time and $\sim 1.5 \mathrm{~s}$ decay time. (c) 2004 American Institute of Physics. [DOI: 10.1063/1.1688456]
\end{abstract}

Photonic molecular devices have attracted tremendous interests because of their small size and light weight. The biologic protein bacteriorhodopsin (bR) and its derivatives are among the most promising candidates for potential applications in biomolecular photonics due to their unique properties. ${ }^{1-3}$ Bacteriorhodopsin is the light harvesting protein found in the purple membrane of halobacterium halobium and is closely related to the visual pigment rhodopsin. It displays a characteristic broadband absorption profile in the visible spectral region. After absorbing photons, the molecules undergo several structural transformations in a welldefined photocycle. It exhibits a large optical absorption cross section and nonlinearity, ease of optimization and tailoring optical properties, and high stability toward photodegradation and temperature. Bacteriorhodopsin has been proposed for a wide range of applications. ${ }^{4-10}$ Among them, photonic switching has been investigated extensively. ${ }^{10-13}$ Most of the previous works on photonic switching were based on the absorption change of $\mathrm{bR}$ after illumination. The wavelength is limited to the absorption peak of the ground $\mathrm{Br}$ state at $\lambda=570 \mathrm{~nm}$ and excited $M$ state at $\lambda=412 \mathrm{~nm} \cdot{ }^{10,12}$ Moreover, the contrast ratio of such devices is in the 300:1 range, which is inadequate for practical applications. ${ }^{14}$

In this letter, we demonstrate a photoactivated switching with good dark state and high contrast at $\lambda=660 \mathrm{~nm}$ based on the photoinduced birefringence in the bR film. Photoinduced optical anisotropy is one of bR's inherent attributes. It is regarded as a result of the orientation of bacteriorhodospin molecules in a statistical view. Initially, the bR film is isotropic owing to the random distribution of the anisotropic photosensitive molecules. When the film is illuminated by a linearly polarized light, those $\mathrm{bR}$ molecules best aligned along the light polarization direction are bleached while those with

${ }^{a)}$ Electronic mail: swu@mail.ucf.edu perpendicular orientation are spared, leading to the macroscopic optical anisotropy. Generally speaking, the photoinduced anisotropy contains photoinduced dichroism and photoinduced birefringence. The photoinduced dichroism reaches the maximum when the probing wavelength is around the absorption peak and becomes zero when the probing wavelength is far from the absorption resonance. Here, we investigate the photoinduced anisotropy at $\lambda=660 \mathrm{~nm}$, which is far from the absorption resonance of the $\mathrm{Br}$ and $\mathrm{M}$ states, in order to avoid the photoinduced dichroism and to extend its application toward long wavelengths.

In our experiment, the biological molecules bR were doped in polyvinyl alcohol film. The sample films were purchased from Munich Innovative Biomaterials $\mathrm{GmbH}$ (order number WT1N3). Its thickness is $\sim 80 \mu \mathrm{m}$ and optical density is 3 at $\lambda=570 \mathrm{~nm}$. Figure 1 shows the experimental apparatus. A diode laser beam with $\lambda=660 \mathrm{~nm}$ was used to probe the sample. The sample was placed between two crossed polarizers $\mathrm{P} 1$ and $\mathrm{P} 2$. P1 and P2 are Glan-Thompson polarizers (Edmund Industrial Optics) with $10^{6}$ extinction ratio. A linearly polarized diode-pumped Nd-YAG laser with $\lambda=532 \mathrm{~nm}$ was used as a pumping beam. A visible broadband quarter-wave plate (Newport 10RP44-1) and a linear

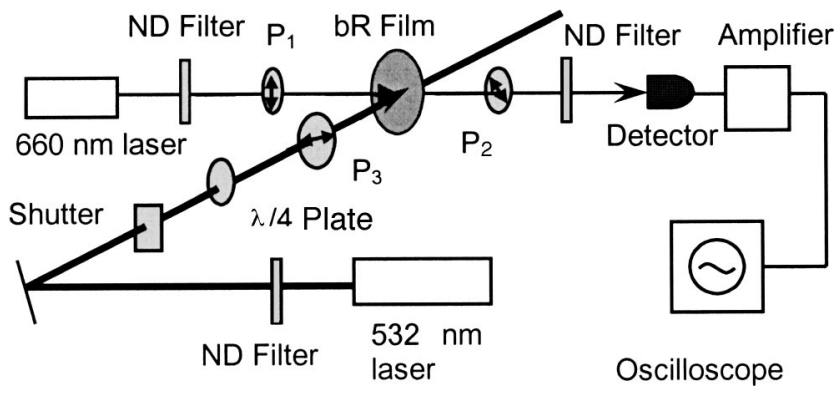

FIG. 1. Experimental apparatus for investigating photoinduced birefringence and photonic switching using a bR film. $\mathrm{P}_{1}, \mathrm{P}_{2}$, and $\mathrm{P}_{3}$ are polarizers: $\mathrm{P}_{1}$ and $\mathrm{P}_{2}$ are crossed, $\mathrm{P}_{3}$ is at $45^{\circ}$ with respect to $\mathrm{P}_{1}$. 


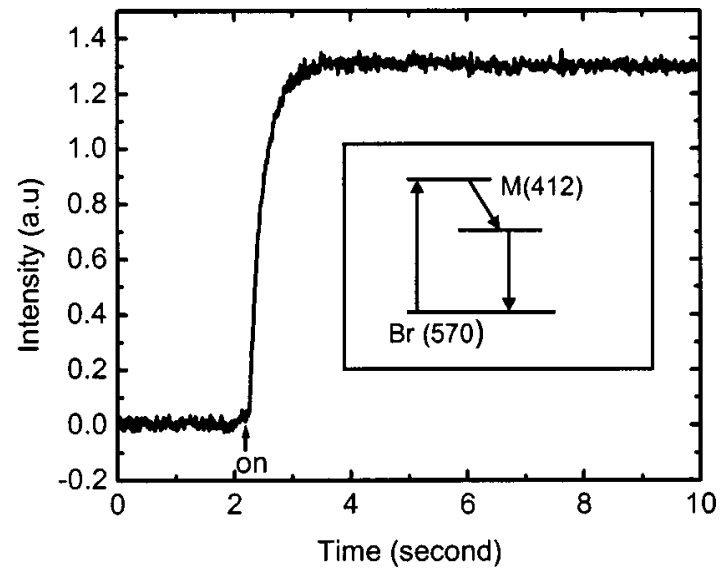

FIG. 2. Experimental results before and after the pumping beam is turned on. The inset depicts the photocycle and the corresponding three level transition states.

polarizer P3 were added to the pumping beam path for selecting the desired polarization state. In our experiment, the optical axis of the quarter-wave plate is oriented at $45^{\circ}$ with the respect to the polarization direction of the $532 \mathrm{~nm}$ laser to transfer linearly polarized light to circularly polarized light. $\mathrm{P} 1$ is oriented at $45^{\circ}$ with respect to the polarizer P3 in order to obtain maximum phase retardation. As illustrated in Fig. 1, the pumping beam overlaps with the probing beam on the sample.

When the pumping beam is blocked, the probing beam could not reach the detector because the bR film is isotropic. When the pumping beam is turned on, the analyzer P2 transmits some probing light, as shown in Fig. 2. Upon excitation of the initial $\mathrm{Br}$ state, the bR molecule goes through several spectroscopically distinguished intermediate states $(K, L$, $M, N$, and $O$ ) and relaxes back to the initial state spontaneously. ${ }^{15}$ Usually, we may consider only the $\mathrm{Br}$ and $\mathrm{M}$ states since the lifetimes of other intermediate states in the photocycle are much shorter compared to $\mathrm{Br}$ and $\mathrm{M}$ states, as shown in the inset of Fig. 2. The initial $\mathrm{Br}$ state has an absorption peak at $\lambda=570 \mathrm{~nm}$ while the long-lived M state has an absorption peak at $\lambda=412 \mathrm{~nm}$. The pumping beam wavelength at $\lambda=532 \mathrm{~nm}$ is close to the absorption peak. Under the exposure to the pumping beam, the bR molecules are selectively excited. Only those bR molecules oriented with their transition dipole moments in or near the polarization direction of the incident beam absorb the light and transit to the excited state M. As a result, the bR molecules exhibit an anisotropic distribution in the illuminated areas. Such an anisotropic distribution rotates the polarization direction of the probing beam. Therefore, a portion of the probing beam transmits through the crossed analyzer. The transmittance of the system can be described by Jones calculus and is given by: ${ }^{6}$

$$
T=\frac{1}{2} T_{\mathrm{P} 1}\left[T_{e} \exp \left(-j \frac{\Gamma}{2}\right)-T_{o} \exp \left(j \frac{\Gamma}{2}\right)\right] T_{\mathrm{P} 2}
$$

where $T_{\mathrm{P} 1}$ and $T_{\mathrm{P} 2}$ are the transmittance of the $\mathrm{P}_{1}$ and $\mathrm{P}_{2}$ polarizers along their optical axes, and $T_{e}$ and $T_{o}$ are the transmittance of the probing beam along the photoinduced extraordinary and ordinary axis, respectively. The photoinduced phase retardation $\Gamma$ is given by

$$
\Gamma=\frac{2 \pi d}{\lambda_{p}}\left(n_{e}-n_{o}\right)
$$

where $\lambda_{p}$ is the wavelength of the probing beam; $d$ is the thickness of the film; $n_{e}$ and $n_{o}$ are the refractive index corresponding to the film's photoinduced extraordinary and ordinary axes, respectively. Finally, the transmitted probing beam intensity can be obtained by

$$
I_{t}=\frac{1}{4} I_{i} T_{\mathrm{P} 1}^{2} T_{\mathrm{P} 2}^{2}\left\{T_{e}^{2}+T_{o}^{2}+2 T_{e} T_{o}\left[\sin ^{2}\left(\frac{\Gamma}{2}\right)-\cos ^{2}\left(\frac{\Gamma}{2}\right)\right]\right\} .
$$

In Eq. (3), $I_{i}$ is the incident intensity of the probing beam before the polarizer $\mathrm{P}_{1}$. From Eq. (3), the transmitted light intensity through the polarizer $\mathrm{P}_{2}$ is determined by the change in absorption, refractive index, dichroism, and birefringence exhibited in the $b R$ films. Since the dichroism at $\lambda=660 \mathrm{~nm}$ is almost zero, we can assume that $T_{e}=T_{o}$ $=T_{\mathrm{bR}}$. Under such a circumstance, Eq. (3) is reduced to

$$
I_{t}=I_{b} \sin ^{2}\left(\frac{\Gamma}{2}\right),
$$

where $I_{b}=I_{i} T_{\mathrm{P} 1}^{2} T_{\mathrm{P} 2}^{2} T_{\mathrm{bR}}^{2}$ presents the probing beam intensity after the parallel polarizers $P_{1}$ and $P_{2}$. Using Eq. (4) and the measured data, we can calculate the photoinduced phase retardation and photoinduced birefringence of the bR film.

Next, we investigate the influence of the pumping and probing beam intensity on the photoinduced birefringence. Results are shown in Fig. 3. Figure 3(a) shows the measured phase retardation of the probing beam as a function of probing beam intensity when the pumping beam was set at $I$ $=500 \mathrm{~mW} / \mathrm{cm}^{2}$. As the probing beam intensity increases, the phase retardation of the probing beam decreases. Although $\lambda=660 \mathrm{~nm}$ is far away from the resonance absorption of the $\mathrm{Br}$ and $\mathrm{M}$ states, it still exhibits a small absorption in the $\mathrm{Br}$ state. When the probing intensity is weak, only few molecules in the $\mathrm{Br}$ state are excited to the $\mathrm{M}$ state by absorbing the $\lambda=660 \mathrm{~nm}$ photons. Most of the molecules in the ground state are bleached to the excited state by the high intensity green pumping beam. As the probe intensity increases, its contribution to the $\mathrm{Br}$ state bleaching increases. Since the polarization direction of the probing beam is at $45^{\circ}$ relative to the pumping beam polarization, the anisotropic distribution of bR molecules in the film is reduced, leading to the decreased photoinduced anisotropy.

Figure 3(b) shows the phase retardation of the probing beam as a function of pumping beam intensity when the probing beam was set at $5 \mathrm{~mW} / \mathrm{cm}^{2}$. The phase retardation increases linearly with the pumping beam intensity in the low intensity regime and gradually saturates as the pumping beam intensity gets higher than $\sim 500 \mathrm{~mW} / \mathrm{cm}^{2}$. It is easy to understand this phenomenon. When the pumping beam is weak, the number of the excited molecules is proportional to the intensity of the pumping beam. Certainly, the photoinduced phase retardation is proportional to the pumping beam intensity. However, in the high intensity regime, all the molecules in the ground state have been bleached to the excited state and no more molecules are spared for the increased 

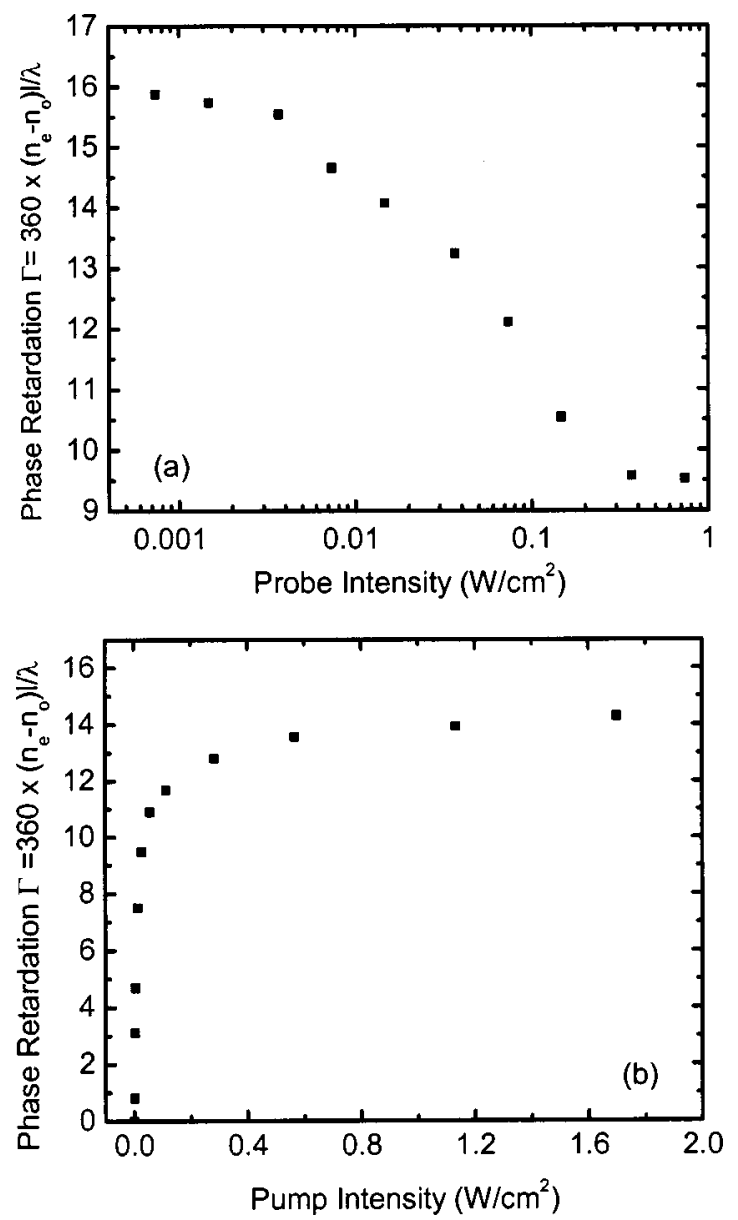

FIG. 3. The induced birefringence as a function of pump intensity (a) and probe intensity (b).

pumping beam. As a result, the phase retardation is saturated. The photoinduced birefringence was estimated to be $n_{e}$ $-n_{o} \approx 3.2 \times 10^{-4}$.

A photonic switching with high contrast ratio (CR) can be obtained if the pumping intensity is $>500 \mathrm{~mW} / \mathrm{cm}^{2}$ and the probing intensity is $<5 \mathrm{~mW} / \mathrm{cm}^{2}$. In our experiment, we achieved CR 1000:1 using $500 \mathrm{~mW} / \mathrm{cm}^{2}$ pumping beam intensity and $5 \mathrm{~mW} / \mathrm{cm}^{2}$ probing beam intensity. As shown in Fig. 2, the photodiode detector has a small thermal noise around the baseline. We have taken the average reading of the noise from the voltmeter while calculating the contrast ratio. High contrast ratio is particularly desirable for photoactivated switching applications.

Figure 4 demonstrates such a photonic switching using our bR film. The response speed is slow due to the long lifetime of the M state $(\sim 10 \mathrm{~s})$. The M-state lifetime can be easily improved by changing the $p \mathrm{H}$ value and humidity during fabrication process. From Eqs. (2) and (4), the transmit-

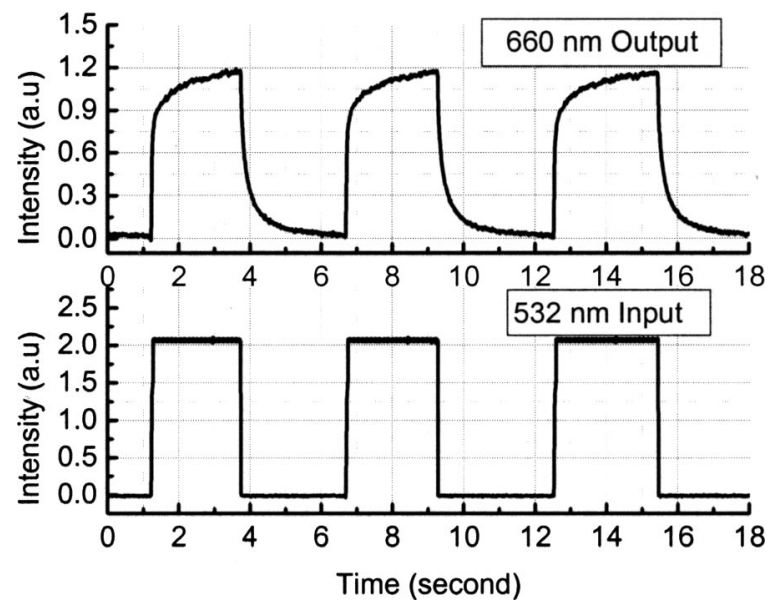

FIG. 4. The measured optical switching results. Pump beam: $\lambda=532 \mathrm{~nm}$ with $I=500 \mathrm{~mW} / \mathrm{cm}^{2}$. Probe beam: $\lambda=660 \mathrm{~nm}$ with $I=5 \mathrm{~mW} / \mathrm{cm}^{2}$.

tance can be largely enhanced by increasing the film thickness. If the bR film thickness is increased to $d \sim 1 \mathrm{~mm}$, then $\Gamma=\pi$ can be induced and high transmittance obtained.

In conclusion, we have demonstrated a photonic switching using a bacteriorhodopsin polymer film. The device exhibits a $\sim 1000: 1$ contrast ratio and the response time is $\sim 0.6 \mathrm{~s}$ due to the long lifetime of the M state. The M-state lifetime can be shortened by changing the $p \mathrm{H}$ value and humidity during fabrication process.

The UCF group is supported by DARPA under Contract No. DAAD19-02-1-0208 and the Fudan group is supported by the National Science Foundation of People's Republic of China under Grant No. 30100034.

${ }^{1}$ K. Clays, S. V. Elshocht, and A. Persoons, Opt. Lett. 25, 1391 (2000).

${ }^{2}$ P. Bhattacharya, J. Xu, G. Váró, D. L. Marcy, and R. R. Birge, Opt. Lett. 27, 839 (2002).

${ }^{3}$ Y. Okada-shudo, Proc. SPIE 4461, 138 (2001).

${ }^{4}$ T. Okamoto, I. Yamaguchi, and K. Yamagata, Opt. Lett. 22, 337 (1997).

${ }^{5}$ G. E. Dovgalenko, M. Klotz, G. J. Salamo, and G. L. Wood, Appl. Phys. Lett. 68, 287 (1996).

${ }^{6}$ D. Sánchez-de-la-Llave and M. A. Fiddy, Appl. Opt. 38, 815 (1999).

${ }^{7}$ M. Sanio, U. Settle, K. Anderle, and N. Hampp, Opt. Lett. 24, 379 (1999).

${ }^{8}$ R. E. Peale, A. B. Ruffin, and J. E. Donahue, Appl. Opt. 36, 4446 (1997).

${ }^{9}$ X. Tian, O. Matoba, Y. Okada-Shudo, M. Ide, T. Shimura, and K. Kuroda, Appl. Opt. 40, 2310 (2001).

${ }^{10}$ T. Zhang, C. Zhang, G. Fu, L. Gu, G. Zhang, Q. W. Song, B. Parsons, and R. R. Birge, Opt. Eng. (Bellingham) 39, 527 (2000).

${ }^{11}$ P. Ormos, L. Fábián, L. Oroszi, E. K. Wolff, J. J. Ramsden, and A. Dér, Appl. Phys. Lett. 80, 4060 (2002).

${ }^{12}$ C. P. Singh and S. Roy, Opt. Commun. 216, 55 (2003).

${ }^{13}$ P. Wu, D. V. G. L. N. Rao, B. R. Kimball, M. Nakashima, and B. S. DeCristofano, Appl. Phys. Lett. 81, 3888 (2002).

${ }^{14}$ R. R. Birge, N. B. Gillespie, E. W. Izaguirre, A. Kusnetzow, A. F. Lawrence, D. Singh, S. Q. Wang, E. Schmidt, J. A. Stuart, S. Seetharaman, and K. J. Wise, J. Phys. Chem. B 103, 10746 (1999).

${ }^{15}$ R. R. Birge, Annu. Rev. Biophys. Bioeng. 10, 315 (1981). 